\title{
Enseñanza del cuidado enfermero en la dimensión espiritual
}

\author{
Care Nurse Education In The Spiritual Dimension \\ ${ }^{1}$ Efigenia Santa Cruz R. ${ }^{a}$ y ${ }^{1}$ Tania Muro C. ${ }^{a}$
}

\section{RESUMEN}

La presente investigación cualitativa, con enfoque de estudio de caso tuvo como objetivo comprender cómo es la enseñanza del cuidado enfermero en la dimensión espiritual de las estudiantes de enfermería en la Universidad Nacional Pedro Ruiz Gallo, Lambayeque 2014. La población estuvo conformada por las enfermeras docentes, saturándose en siete, recolectándose los datos por entrevista abierta a profundidad, analizándose temáticamente, generándose tres categorías: Fundamentando de la Enseñanza del cuidado enfermero a lo intrínseco y religioso, el ser enfermero: formación en el cuidado de la dimensión espiritual, el hacer enfermero en el cuidado de la dimensión espiritual. En las consideraciones finales, se concluyó que la enseñanza del saber enfermero en el cuidado de la dimensión espiritual se ha visto reducido a casos de enfermería en relación aspectos religiosos, y muchas veces confundido con la dimensión emocional de la persona.

Palabras clave: cuidado enfermero, dimensión espiritual y enseñanza de la dimensión espiritual.

\begin{abstract}
This qualitative research, with a focus on case study aimed to understand how the teaching of nursing care in the spiritual dimension of nursing students in the Lambayeque Pedro Ruiz Gallo National University 2014. The population consisted of nurse educators, saturating in seven, collecting data from open-depth interviews, analyzed thematically, generating three categories: basing teaching care nurse and the intrinsic religious, the nurse being: training care spiritual dimension. In the final considerations , it was concluded that the teaching of nursing knowledge in the care of the spiritual dimension has been reduced to nurse case concerning religious aspects, and often confused with the emotional dimension of the person.
\end{abstract}

Keywords: nurse caring, spiritual dimension, teaching care nurse in spiritual dimension.

'Universidad Nacional Pedro Ruíz Gallo. Lambayeque, Perú.

${ }^{a}$ Licenciada en Enfermería 


\section{INTRODUCCIÓN}

El cuidado humano es comprendido en este estudio como la esencia de la praxis de enfermería, desarrollado de forma procesual en la vivencia humana y aprendido como competencia profesional mediante el compromiso con 1o humano en su integralidad cuerpo-mente-alma, abrazando la ética, moral y estética, fomentadores de una postura profesional capaz de contemplar las múltiples dimensiones de la persona humana a partir de la transversalidad entre el conocimiento científico y el ser humanístico. En este sentido, las directrices curriculares de formación en enfermería definen que la educación superior del enfermero debe primar por el desarrollo de competencias y habilidades humanistas, crítico reflexivas con las dimensiones biopsicosociales espirituales del ser humano, dotando a su praxis de sentido de responsabilidad social por la salud integral del otro y de sí (autocuidado físico y mental), en búsqueda de la calidad, eficiencia y resolutividad en el cuidado de las vidas humanas (Alonso y Escorcia, 2003, 3-8).

En esa dirección, se discule también la formación pedagógica del docente de enfermería para atender las nuevas demandas educacionales de la sociedad, constatándose que no es posible formar enfermeros generalistas, críticos y reflexivos sin que los enfermeros profesores tengan una adecuada formación (Marriner, 2006). De este modo, se desprende que la praxis de enfermería debe transcender a la acción técnica e ir al encuentro de la acción responsable por el crecimiento mutuo de quien cuida y de quien es cuidado.

Formar para el cuidado transpersonal requiere el rescate del significado de la existencia humana, el cual solamente podrá ser alcanzado cuando sentido y vivido por el amor y respeto a la dignidad de la vida y de la sensibilidad a su existencia (Alonso y Escorcia, 2003, 3-8). Lo que motivo investigar el proceso de enseñar el cuidado enfermero en la dimensión espiritual en la Facultad de Enfermería de la Universidad Nacional Pedro Ruiz Gallo en los semestres Académicos I - II - 2013, a través de las actividades teóricas prácticas, en sus etapas de planificación, ejecución y evaluación, con el objetivo de comprender como es la enseñanza del cuidado enfermero en la dimensión espiritual.

Como parte del cuidado, todo profesional de enfermería debe ofrecer, el cuidado espiritual. Para lograr esta competencia, las instituciones formadoras deben planificar ejecutar y evaluar actividades curriculares que permitan lograrla. Además se observa que existen debilidades en relación a la enseñanza del cuidado en esta dimensión, evidenciándose en la práctica de la enfermería de nuestras egresadas, quedando el cuidado de la dimensión espiritual de la persona cuidada meramente en el marco teórico y conceptual de autoridades, docentes y estudiantes, lo cual no se manifiesta en el hacer de la egresada, reduciéndose las necesidades espirituales al aspecto religioso.

En esencia la espiritualidad es un fenómeno íntimo y trascendente que es vital para la recuperación de calidad del paciente, además una fortaleza, motivación y búsqueda del significado de la vida. Es por esto que el cuidado espiritual ofrecido por el profesional de enfermería debe ser con un sentido de responsabilidad, respeto y dedicación al paciente, sin embargo poco se conoce al respecto, por no existir investigaciones que aborden y hallan profundizado en el cuidado 
espiritual a las personas cuidadas, puesto que muchas veces se olvida que la persona como ser humano posee dimensiones que intervienen y forman parte en su salud y bienestar dejando esto para los sacerdotes, consejeros espirituales e incluso también para psicólogos.

La relevancia de este estudio es mostrar la realidad de la formación superior de enfermería en lo que se refiere al desarrollo de habilidades psicoafectivas y cognitivas para la satisfacción de necesidades espirituales que se relacionan con el proceso salud enfermedad. La escasez de estudios que abordan esta temática en las bases de datos investigadas refuerza y justifica su importancia, de modo a constituirse como un problema a ser revelado.

\section{MATERIALES Y MÉTODOS}

Esta investigación, cualitativa con abordaje de Estudio de Caso, tuvo como escenario la Facultad de Enfermería de la Universidad Nacional Pedro Ruiz Gallo, Lambayeque 2014 y la muestra estuvo conformado por las docentes encargadas de enseñar el cuidado enfermero, vale decir, de las asignaturas de carrera Enfermería Básica I y II, Enfermería en Salud del Adulto y Anciano I y II, Enfermería Materno Perinatal, Enfermería en Salud del Niño y Adolescente. La muestra fue determinada por saturación. Recolectándose los datos, por entrevista abierta a profundidad y su tratamiento a través del análisis temático, las fases fueron: Análisis textual: el discurso se centra en su textualidad; para ello utiliza la descripción, aplicada a los discursos no verbales, y la transcripción, aplicada a los discursos orales. Análisis contextual, permite la interpretación del discurso. En la descripción o a que transcripción se incluyeron todos aquellos elementos antecedentes y del contexto que puedan contribuir a su interpretación. Por otro lado, en la transcripción se incluyó todas las incidencias no verbales (silencios y su duración, modulaciones y énfasis utilizados, gestos significativos de la expresión, entre otras). La descripción, debe ser detallada e incluir todos los elementos de la situación que la configuran como discurso.

El análisis de contenido consiste básicamente en la descomposición o fragmentación del texto en unidades constitutivas para su posterior codificación según un sistema de categorías, generalmente preestablecido.

Se aplicaron los principios éticos de Belmont Report, de beneficencia, respeto a la dignidad de la persona y la justicia (Polit y Hungler, 2000, 50-60). Para el soporte científico se tuvo en cuenta los principios de cientificidad de credibilidad y auditabilidad (Morse, 2003, 3450).

\section{RESULTADOS Y DISCUSIÓN}

Las enfermeras fueron entrevistadas sobre el proceso de enseñanza del cuidado enfermero en las experiencias teórico prácticas. Las experiencias teóricas referidas a las actividades de enseñanza aprendizaje que se realizan en el aula, abordando desde el marco teórico conceptual y filosófico de la enfermería así como el cuidado enfermero, utilizando diversas metodologías todo ello bajo un paradigma de transformación. A continuación se presentan las categorías y sub categorías que fueron emergiendo a lo largo de la investigación. 


\section{Fundamentando la enseñanza del cuidado enfermero a lo intrínseco y religioso}

El saber enfermero en el cuidado de la dimensión espiritual, es el conjunto de conocimientos respecto a la espiritualidad, necesidades espirituales, bienestar y distrés espiritual y el cuidado de esta dimensión. Donde se integra el conocimiento acumulado, el conocimiento relacionado con la fuente y las terapias disponibles para cl cuidado de la espiritualidad, logrando desarrollar un plan de cuidado individualizado y de alta calidad que emergen de las siguientes subcategorías:

\subsection{Derivando el cuidado espiritual a la práctica religiosa}

Con frecuencia el profesional de enfermería reduce el concepto de espiritualidad a la religión. Ante esto, es importante que la espiritualidad de las estudiantes se clarifique y se reconozcan los atributos de este concepto, para delinear un plan de cuidado que satisfaga las necesidades espirituales y así proveer un cuidado holístico. Castro F. (2012) afirma que se puede mostrar colérico, por creer que es injusto lo que está sucediendo y por lo mucho de lo sucedido en el pasado, y sobre todo un sentimiento desolador de vacío.

Utilizó casos de enfermería y discuto en aula. Les digo a las estudiantes cuando la persona está en agonía indicar a los familiares a pedirles perdón y a perdonarlos: Se han dado casos en que el paciente ha estado grave, en D...I

La estudiante debe aprender a reconocer que las personas cuidadas tienen la necesidad de liberarse de la culpa, perdonarse y ser perdonado, ante la proximidad a la muerte.
Además reconocen que el perdón a Dios, asimismo y a los demás es imprescindible para generar tranquilidad, paz y oportunidad para iniciar una nueva vida.

\subsection{Enseñando a reconocer la importancia del cuidado enfermero en la dimensión espiritual} de la persona

La dimensión espiritual, representa la más noble y trascendente de las dimensiones de la persona. Es subjetiva, compleja e intangible, permea y vitaliza las otras dimensiones; de manera que todos los valores, pensamientos, comportamientos, decisiones, experiencias y preocupaciones están relacionados con esta. La espiritualidad es parte fundamental en el cuidado de enfermería, pues en ella se origina el significado, el propósito y plenitud en la vida, el deseo de vivir, creencias y la fe en su ser, en otros y en Dios (Sánchez, 2004).

Es importante el cuidado de la dimensión espiritual, de la persona que está enferma o a la misma familia se le orienta a tratar la parte espiritual, guio a la estudiante para que le de conformidadalapersona D...2

Es una cualidad o esencia que integra o transciende la naturaleza biopsicosocial del individuo. Es todo aquello que le da significado a la vida. Implica una cualidad de trascendencia, una fuerza que guía, algo fuera del ser, más allá del individuo. Son creencias o sistema de valores que provee fuerzas, esperanza y significado a la vida. 


\subsection{Enseño a identificar la necesidad de liberarse de la culpa, y a perdonarse}

La persona con problemas de salud siente miedo a no ser escuchado, a no poder expresar sentimientos y temores, intenta buscar alguna explicación o sentido al sufrimiento y se pregunta: ¿por qué a mí? Pero en recordar su vida pasada a veces surge la culpa por no haberse cuidado, que si no es bien elaborada, puede ser generadora de un distrés espiritual, "se puede mostrar colérico, por creer que es injusto de lo que está sucediendo y por lo mucho de lo sucedido en el pasado, y sobre todo un sentimiento desolador de vacío"

Utilizó casos de enfermeria y discuto en aula. Les digo a las estudiantes cuando la persona está en agonía indicar a los familiares a pedirles perdón y a perdonarlos: Se han dado casos en que el paciente ha estado grave, en D...1

La estudiante debe aprender a reconocer que las personas cuidadas tienen la necesidad de liberarse de la culpa, perdonarse y ser perdonado, ante la proximidad a la muerte. Además reconocen que el perdón a Dios, a sí mismo y a los demás es imprescindible para generar tranquilidad, paz y oportunidad para iniciar una nueva vida.

\section{El Ser enfermero: formación en el cuidado de la dimensión espiritual}

El ser enfermera significa forzar el intelecto para estar al día con el avance del conocimiento, es analizar ese conocimiento para aplicarlo en el momento justo, significa forzar el alma para entrar en contacto con las necesidades de otros (Alarcón, 2009).
Es importante que el estudiante comprenda su propia espiritualidad para entender de manera respetuosa las necesidades espirituales de la persona que cuida. Así también, es necesario que tenga la espiritualidad y religión como conceptos de vida; pues ello le permitirá cuidar a la persona sana o enferma, con situaciones estresantes y problemas de toda índole en su laborar, proporcionándole fortaleza interior (Benito et al, 2011).

\subsection{El ser enfermero fundamentado en la existencia de un ser superior.}

Las estudiantes deben aprender a considerar a las personas como seres dinámicos, en crecimiento y espirituales que anhelan estar conectados con otros. La espiritualidad le permite a la persona estar ligado con una fuente eterna de bondad, misterio, vida, creatividad y serenidad. Esta se basa en la trascendencia o compromiso religioso que se da a través de la conexión con un ser superior. En ese sentido, esta conexión ayuda a dar tranquilidad, paz, ánimo, gozo espiritual, confianza, protección y cuidado (Pinto, 2007).

Cuando el caso de enfermería lo amerita les digo a las estudiantes hay alguien superior a nosotros que nos protege, en el cual nos debemos encomendar para que nos de fuerza. D...4

La fuerza superior se devela como una dimensión sagrada del ser humano, como una fuerza que motiva e impulsa su vida. En la formación de estudiantes la espiritualidad le servirá de apoyo y de seguridad y se traducirá en el cuidado que ella tenga consigo mismo y para con los demás. 
Es crucial la actitud que la enfermera tenga ante el sufrimiento. La filosofía o sentido de la vida y creer en algo o alguien, influyen en el modo de relacionarnos con los que sufren (Beca, 2008).

\subsection{Iniciándoles en el cuidado de la dimensión espiritual}

Los estudiantes de enfermería deben aprender a cultivar su ser para estar preparados para asistir, entender, acompañar y ayudar a la persona enferma. Pues el sufrimiento que este proceso conlleva es una experiencia humana tremendamente compleja, que siempre necesita de ellos. Se requiere considerar a la persona como única, individualizando el cuidado; siendo imprescindible la empatía y dedicación para obtener el mayor bienestar y confort (Torralba, 2006).

Ustedes van a dar apoyo espiritual; y que aunque tengan la ayuda del sacerdote, serán ustedes las que están mayor tiempo en contacto directo con el sufrimiento de la persona para hablarles de Dios y asi procurar su bienestar espiritual. D...3

La enfermera desempeña un papel fundamental en el cuidado y tratamiento de la persona enferma. En el entorno hospitalario es la enfermera quien más tiempo permanece cuidando a la persona y a la familia, por lo tanto es la que más conoce y puede aportar datos para su mejor control sintomatológico y brindar cuidado psicológico y espiritual (Torralba, 2006).

\section{EI hacer enfermero durante la formación en el cuidado de la dimensión espiritual}

El hacer enfermero se refiere al desarrollo de acciones contempladas en el ejercicio disciplinar; que abarca la promoción de la salud, la prevención de la enfermedad, el tratamiento oportuno y la rehabilitación del sujeto de cuidado (Torralba, 2006).

\subsection{Enseño a cuidar la necesidad de ser reconocido como persona}

La identidad del ser humano procede igualmente de los otros, de la representación que ellos se hacen. De esta manera, es importante la satisfacción de la necesidad de ser reconocido, como persona comprende acciones que le permitan sentirse querida y se reconozca su valía personal a través de acciones como: el saludo, ser nombrado, recibir un trato amable, mostrar interés en su confort e imagen corporal, cuidar los gestos, tacto terapéutico, escucha activa, sentirse asociado a las decisiones terapéuticas y que sean respetadas sus diferencias y características intrínsecas (Núñez, 2010).

Deben llamar a las personas por su nombre, siempre con mucho respeto, en ningún momento deben llamarlos por el número de cama o algún sobrenombre" D...3

Es importante llamar a la persona por su nombre puesto que la despersonalización en el cuidado diario de enfermería lleva al anonimato y en consecuencia, la persona se sentirá reducida o identificada por su enfermedad (San Martín, 2007).

\subsection{Cuidado enfermero dirigido de la necesidad de encontrar sentido a la vida}

Las enfermeras brindan a la persona información acerca de su estado de salud, evolución y tratamiento; a pesar de no saber que así satisfacen la necesidad de ser reconocido como persona; puesto que es su derecho conocer todo lo referente a su condición de salud y garantizar el 
respeto de su dignidad humana. Brindarle información clara, comprensible y precisa, permitirá fortalecer su autonomía para tomar decisiones en su cuidado.

Cuando la persona nos dice que son una carga para su familia, que prefieren morir, reniega por su situación y a veces se porta agresivo con su familiar. La enfermera debe conducirla a que reflexione sobre su forma de pensar y actuar, que trate de ser objetivo y valore el apoyo familiar que le están brindando. D...7

Se recomienda al estudiante que es importante no reducir el cuidado a la tecnología o procedimental, porque lo alcja de un cuidado holístico, el cual va más allá del curar, busca la sanación. La curación ocurre cuando se detiene o se revierte el proceso de la enfermedad; la sanación, supone un proceso más complejo, puede o no incluir la cura. La sanidad integra cuerpo, mente y espíritu, y se alcanza cuando la espiritualidad es incorporada en el cuidado enfermero.

\section{CONCLUSIONES}

La enseñanza del saber enfermero en el cuidado de la dimensión espiritual se ha visto reducido a casos de enfermería en relación aspectos religiosos, y muchas veces confundido con la dimensión emocional de la persona. Así también, las estudiantes deben reconocer la importancia que tiene el fortalecimiento de la espiritualidad ante la enfermedad. El ser enfermero en el cuidado de la dimensión espiritual, se orienta a las creencia en un ser superior y en sus prácticas religiosas.
El hacer enfermero en el cuidado de la dimensión espiritual es realizado de forma empírica, porque las estudiantes desconocen la existencia de las necesidades espirituales. Las intervenciones que ellas realizan han podido ayudar a las personas en su espiritualidad, pero no son conscientes de haberlo hecho.

Capacitar a las docentes para la enseñanza de la dimensión espiritual a las estudiantes durante la formación.

\section{AGRADECIMIENTOS}

A las docentes de Facultad de Enfermería por participar en el estudio.

A la Universidad Nacional de Jaén por la oportunidad que nos brinda para poder difundir esta investigación.

\section{REFERENCIAS BIBLIOGRÁFICAS}

Alarcón I. 2009. Necesidades espirituales de los pacientes terminales del servicio de medicina en el Hospital Nacional Dos de Ma y o. UR L d is ponible en : http://www.cybertesis.edu.pe/sisbib/2009/alarc on_si/pdf/alarcon_si.pdf (consultado el 20 de Octubre de 2012).

Alonso, M. y Escorcia I, 2003. El ser humano como una totalidad. Salud Uninorte 17: 3-8.

Beca J. 2008. El cuidado espiritual del enfermo como responsabilidad del profesional de la salud. Ética de los Cuidados. URL disponible en: http://www.indexf.com/eticuidado/n1/et6734.php (consultado el 25 de noviembre del2012). 
Benito E, Barbero J y Payás A. 2011. El acompañamiento espiritual en cuidados paliativos. URL disponible en: http://hospicemadreteresa.org.ar/esp/wpcontent/uploads/2011/11/guia-espiritualsecpal.pdf (consultado el 19 de Noviembre del 2012).

Castro F. 2012. Aspectos emocionales y espirituales de la enfermedad: "Sufrir con los ojos abiertos". URL disponible en: Chttp://archimadrid.es/dpsanitaria/rc/esponc.pd f(consultado el 15 de Octubre del 2012).

Marriner A. Modelos y Teorías de Enfermería. 6ta ed. España: Mosby y Doyma;2006

Morse, M. 2003. Emerger de los datos: los procesos cognitivos del análisis en la investigación cualitativa. Colombia: Universidad de Antioquia.

Núñez P. 2010. La espiritualidad en el paciente oncológico: una forma de nutrir la esperanza $y$ fomentar un afrontamiento positivo a 1 a e $n$ fermedad termina 1 . U R L dis pon ible e n : www.scielo.org.bo/pdf/rap/v10n1/v10n1a5.pdf (consultado en el 15 de Octubre del2012).

Pinto N. 2007. Bienestar espiritual de los cuidadores familiares de niños que viven enfermedad crónica. Redalyc Sistema de Información Científica, URL disponible en: http://redalyc.uaemex.mx/src/inicio/ArtPdfRed .jsp? $\mathrm{iCve}=145212858003$ (consultado el 20 de Octubre del 2012).
Polit, D. y Hungler B. 2000. Investigación Científica en Ciencias de la Salud. México: Mc Graw-Hill Interamericana.

Sánchez B. 2004. Dimensión Espiritual del Cuidado de Enfermería en Situaciones de Cronicidad y Muerte. URL disponible en: http:/www.bdigital.unal.edu.co/2050/1925/978 9587611335.pdf (consultado el 5 de Octubre del 2012).

San Martín C. 2007. Espiritualidad en la Tercera E d a d. U R L d i s p o n i b l e e n : http://www.palermo.edu/cienciassociales/publi caciones/pdf/Psico 8/8 P s i co\%2008. (Consultado el 18 de Octubre del 2012).

'lorralba, F. 2006. Necesidades espirituales del ser humano. URL disponible en:http://www.bizkeliza.org/areas pastorales/salud-y-terceraedad/ artículos/acompanamiento-humano-ypastoral/necesidades-espirituales-del-ser humano-frances-torralba (consultado el 25 de noviembre del 2012).

\section{CORRESPONDENCIA}

Efigenia Rosalía Santa Cruz Revilla

Calle 12 de Octubre 150 La Victoria - Chiclayo, Perú.

lia-2066@hotmail.com 